\title{
Design of Power Integration and Line Loss Management System Based on Big Data
}

\author{
Wu Junying ${ }^{1}$, Xin Rui ${ }^{1}$, Yang Huifeng ${ }^{1}$, Zhao Jianbin ${ }^{1}$, Lu Xin ${ }^{1}$, Chen Jing ${ }^{2}$ \\ ${ }^{1}$ State Grid HeBei Information \& Telecommunication Company, HeBei ShiJiaZhuang 050021, China \\ ${ }^{2}$ SGIT-GreatPower, Fujian, Fuzhou, 350003, China
}

Keywords: line loss management, integrated acquisition, big data

\begin{abstract}
In view of the current line loss problem of transmission lines, combined with the current computer technology, an integrated power collection and line loss analysis system is proposed. The article first describes the relationship between the electricity collection and line loss system and other power systems, so as to better clarify the system data sources; on the basis of the above analysis, the functional modules of the system are analyzed, and the data comparison and analysis modules in the monitoring module are highlighted. Finally, the technical solutions and related functions implemented by the system are analyzed in detail, and the display interface is given.
\end{abstract}

With the continuous deepening of China's power system reform work, the line loss rate has become one of the important indicators for judging the comprehensive benefits of power companies, reflecting the work efficiency, operational level and production technology of power companies. In this context, how to accurately manage the line loss has become the primary problem that plagues the management of power companies. At present, there are many factors that cause line loss in electrical enterprises, including power distribution network structure, distribution network technology, equipment status, and line model, transformer performance, and reactive power compensation device. In addition, the quality of power marketing management will also lead to the problem of power line losses. Therefore, in order to achieve accurate management of line loss, power companies need to start from the following two aspects: On the one hand, power companies need to collect data on many line loss factors; on the other hand, power companies are completing data collection. After that, the data needs to be analyzed to obtain the main reason for the line loss and manage it in time. In recent years, under the promotion and implementation of smart meters, power companies have paid more attention to the construction of power information collection systems. At present, a more mature power information collection system has been established. This phenomenon provides greater support for line loss calculation and line loss precision management. Therefore, this paper attempts to construct a line loss management integrated system based on power collection with the electric acquisition system, and design its implementation in detail.

\section{Description of the Relationship between Integrated Acquisition and Line Loss Management Platform and Other Systems}

The system level of the station management platform is mainly composed of data integration and platform integration. Among them, the demand for data integration lies in the integration of power marketing, transportation inspection, scheduling and other systems; platform integration is mainly for a number of data platforms, such as the grid GIS platform, massive data platform, etc. The logical relationship can be expressed as: the electricity collection system stores the collected data into the SCADA system, and the SCADA management system is connected with the marketing management system to facilitate the marketing management system to analyze the electricity, thereby providing a reference for marketing; the data storage platform, through the file access interface at the same time, enables the line loss platform to call the collected power collection data, thereby realizing the line loss calculation for different stations and lines, and combining the calculation results to propose corresponding countermeasures. 


\section{Integrated Function Design of Integrated Acquisition and Line Loss Management System}

The integrated acquisition and line loss management system designed in this paper is mainly composed of four functional modules, including three functions: abnormal monitoring, professional management and business support. among them. The abnormality monitoring module is composed of functions such as data check, indicator evaluation, and measurement failure analysis; professional management is mainly composed of daily line loss calculation, monthly line loss statistics, and indicator management; the business support module includes data interface management and system configuration, log management and other functions. The basic function design of the specific line system is shown in Figure 1.

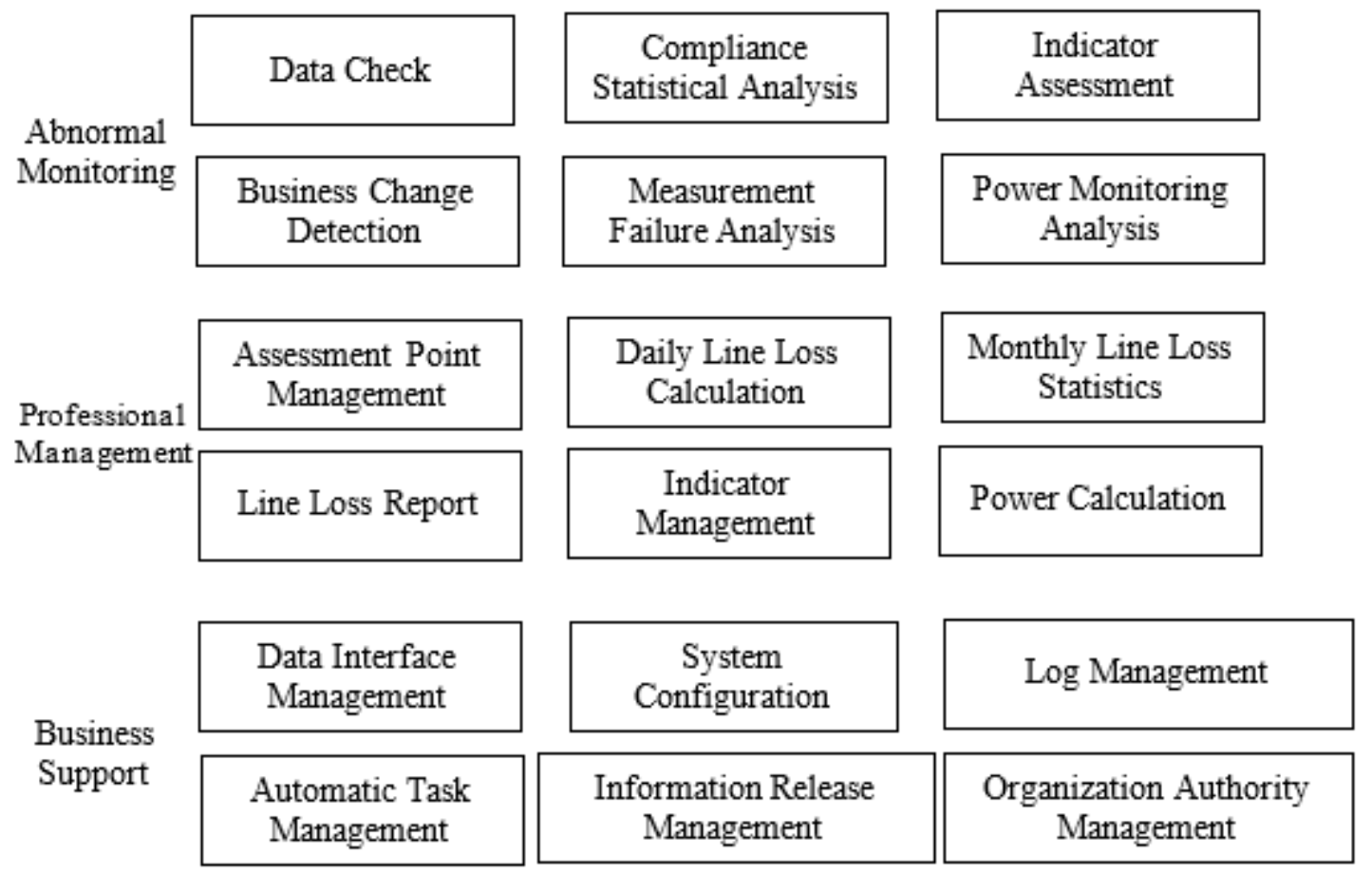

Figure 1 Basic Function Design of the Line System

In the power monitoring analysis in Figure 1, the need to combine line loss, the focus includes the following functions:

First, longitudinal tracing and horizontal correlation analysis. For vertical data tracing, the system can track the data of different calculation results through the system, and find out the minimum required point calculation point through the data trace. The traced data mainly include: this time / the last positive (without) power meter bottom, power; this time / last reverse has (no) power meter bottom, power; meter reading cycle; PT, CT ratio; Date of meter reading, etc. Through the traceability of information, the system summarizes the relevant data that needs to be calculated (such as the original power, theoretical line loss results, "quadruple" line loss results, etc.), and then summarizes the problem.

Second, comparative analysis. Comparative analysis requires the two sets of data, statistical line loss and theoretical line loss, to be calculated separately. After the data is calculated, the results are compared. The system will analyze and compare the system according to different comparison results, and finally draw conclusions. In the comparison process, different basic units are used as comparison objects. For example, a single line is the basic unit, a single low-voltage station area is used as the basic unit, and the main unit is the basic unit, and the power supply area is the basic unit. Through the comparative analysis of different basic unit data, the problem is found, the system finds the source of the problem according to the discovered problem, and implements the preliminary positioning of the problem location.

Third, line loss composition and trend analysis. The fixed loss and the variable loss constitute the theoretical line loss value. The fixed loss and the variable loss in the theoretical line loss value are 
different in proportion and represent different meanings. Through the analysis of different scale values, it can be known whether the current grid operation is normal or not, whether the grid line and the component facilities are operating normally, and the reference data will be provided for the problems occurring during the operation, and the loss reduction scheme is prepared through the reference data. The line loss trend includes three types, namely, ring ratio analysis, year-on-year analysis, and annual line loss changes. The ring ratio analysis refers to the line loss analysis and comparison results of the grid companies in each stage in the same year. By analyzing the comparison results, the actual line loss trend trend chart is obtained. If an abnormal situation is found, the data comparison result is solved in time. Since the line loss trend can monitor the abnormality of the identification, some preset rules can be formulated based on this characteristic. The actual line loss trend chart image is direct, not only can lock the fault problem in time, but also can learn more equipment information. Finally, various line loss data results are calculated through the line loss trend chart, and a comprehensive and detailed graphic chart is given. The line loss changes and the problems appearing are displayed in detail in the graphic chart.

Fourth, the power is intelligently chased. The core of the line loss management system is to collect data according to the existing mathematical model, and to monitor various types of voltage and current in real time through the system, and find abnormal values that do not meet the theoretical results, and find out the abnormal values and The normal values are compared and calculated, and the calculation results are used as a reference for the work of the line-recovery power in order to reduce unnecessary losses. The specific process is: the process of chasing the electricity must start from the category of discriminating the error, so as not to cause misjudgment. Next, according to the judgment, the relevant parameter values of the calculation result are obtained. To analyze the error amount, first analyze the error amount proportional range, and then calculate the corresponding deviation value according to the data model. Unified management is performed by the calculated deviation value, and an early warning prompt is made. The user can query the abnormal situation according to the warning prompt and solve it in time.

Fifth, suspected thief analysis. The suspected thief analysis has a large effect, a wide range of applications, and a comprehensive application object. Through the overall analysis of line loss and overall power, it can provide users with information about stealing electricity and facilitate staff planning and management. The system labels the user information that is suspected of stealing electricity, and focuses on monitoring the user information. The system also provides the user with the information of the line loss information to provide warnings to the user. The system administrator can analyze the existence of the stealing line according to the warning information, and provide information basis for the future investigation of the power stealing behavior. According to the information basis, the scope of the investigation is determined, and the users with the characteristics of the power stealing behavior are regarded as the key investigation targets.

\section{System Application Deployment Design}

In the design of the system application deployment module, taking into account the requirements of the State Grid Corporation for the data integration interface and application of the electrical enterprise, this paper will design the application deployment of the system according to the centralized deployment mode of the network. Table 1 shows the configuration and description of the major hardware devices in a centralized deployment.

\section{Application Realization of the Technical Solution}

The power information acquisition line loss management platform designed in this paper mainly uses the Struts-MVC framework for platform development, and designs the overall system architecture with MVC mode. AJAX technology is used in the design process of the system interface components to give the system rich interface elements and powerful operation functions in B/S mode.

The MVC model is a software design model that originated in the 1980s. This model has become the main mode adopted by Sun in the J2EE platform design process in recent years, and has been 
widely used in various design fields. The main idea of the MVC mode in the application process is to force the input, processing and output parts of the system application to be separated, so that the various parts of the system application can focus on their own tasks, thereby improving the software in the system application.

Table1 Configuration and Description of Major Hardware Devices in Centralized Deployment of the Network

\begin{tabular}{|c|c|c|c|}
\hline $\begin{array}{l}\text { Deployment } \\
\text { location }\end{array}$ & Database & Data type & Use and device requirements \\
\hline & Data storage area & $\begin{array}{l}\text { SAN storage system } 1 \\
\text { set }\end{array}$ & 1. Storage of important power and line loss data \\
\hline & $\begin{array}{l}\text { Document } \\
\text { database }\end{array}$ & SAN Switch (Shared) & $\begin{array}{l}\text { 1. Purpose: Connect storage and platform host } \\
\text { 2. Equipment requirements: Line management system uses } 2 \text { ports (optical } \\
\text { fiber) }\end{array}$ \\
\hline & & $\begin{array}{l}\text { Production database } \\
\text { server }: 1\end{array}$ & $\begin{array}{l}\text { 1. Use: centralized management of station line system data } \\
\text { 2. Equipment requirements: 4CPU (core) } 1.6 \mathrm{GHZ} \text { or more; } 16 \mathrm{G} \text { memory or } \\
\text { more; } 2 \text { Gigabit network card; Unix operating system }\end{array}$ \\
\hline & & $\begin{array}{l}\text { Historical database } \\
\text { server }: 1\end{array}$ & $\begin{array}{l}\text { 1. Use: historical query and marketing query library share; } \\
\text { 2. Equipment requirements: PC server and CPU 3.0GHZ or above }\end{array}$ \\
\hline \multirow[t]{5}{*}{ Jiangxi company } & \multirow[t]{5}{*}{ Core host area } & Application server :2 & $\begin{array}{l}\text { 1. Use: Form an application cluster, deploy an application middleware } \\
\text { software platform, be responsible for the processing of business logic, and run } \\
\text { business applications; }\end{array}$ \\
\hline & & Load balancer :1 & 1. Use: Client's web request load balancing; \\
\hline & & & $\begin{array}{l}\text { 2. Device requirements: The maximum number of concurrent sessions is } \\
\text { greater than 100,000; support SSL, built-in SSL chip acceleration; } \\
\text { double-click switching time is less than } 200 \mathrm{~ms}\end{array}$ \\
\hline & & Stable data (former & 1. Use: Stores previous CAD drawing information \\
\hline & & CAD drawings) & 2. Equipment requirements: PC server is above CPU3.0GHZ \\
\hline
\end{tabular}

An application designed with the MVC pattern will consist of three core components, a model, a view, and a controller. MVC mode has the advantages of fast deployment and high maintainability, as well as the disadvantages of complex development process and not suitable for small and medium-sized applications. However, the emergence of the Struts open source framework can perfectly solve the complex shortcomings of the MVC pattern development process. The Struts open source framework consists mainly of Servlet and JSP tag libraries, which can simplify MVC-based web applications.

To achieve the application platform software, the specific implementation requirements are as follows:

1) Application platform software must pass J2EE certification and only support J2EE 1.4 and above;

2) Supporting many current mainstream technologies, including EJB, Servlet \& JSP, JMS, JTA, etc., and supporting the deployment and construction of various J2EE applications on the basis of various technical applications;

3) The J2EE application platform and the operating system in the system are independent, and can support various operating systems currently on the market, such as UNIX, Linux, etc.;

4) The system supports various heterogeneous hardware and software environments, such as IBM, $\mathrm{HP}$ and other host environments;

5) The database mainly supports the Oracle database;

6) It can support two clusters of single-machine multi-instance and multi-machine multi-instance;

7) It can support Chinese kanji inner code and double-byte encoding. 


\section{Line Loss Analysis Module Design}

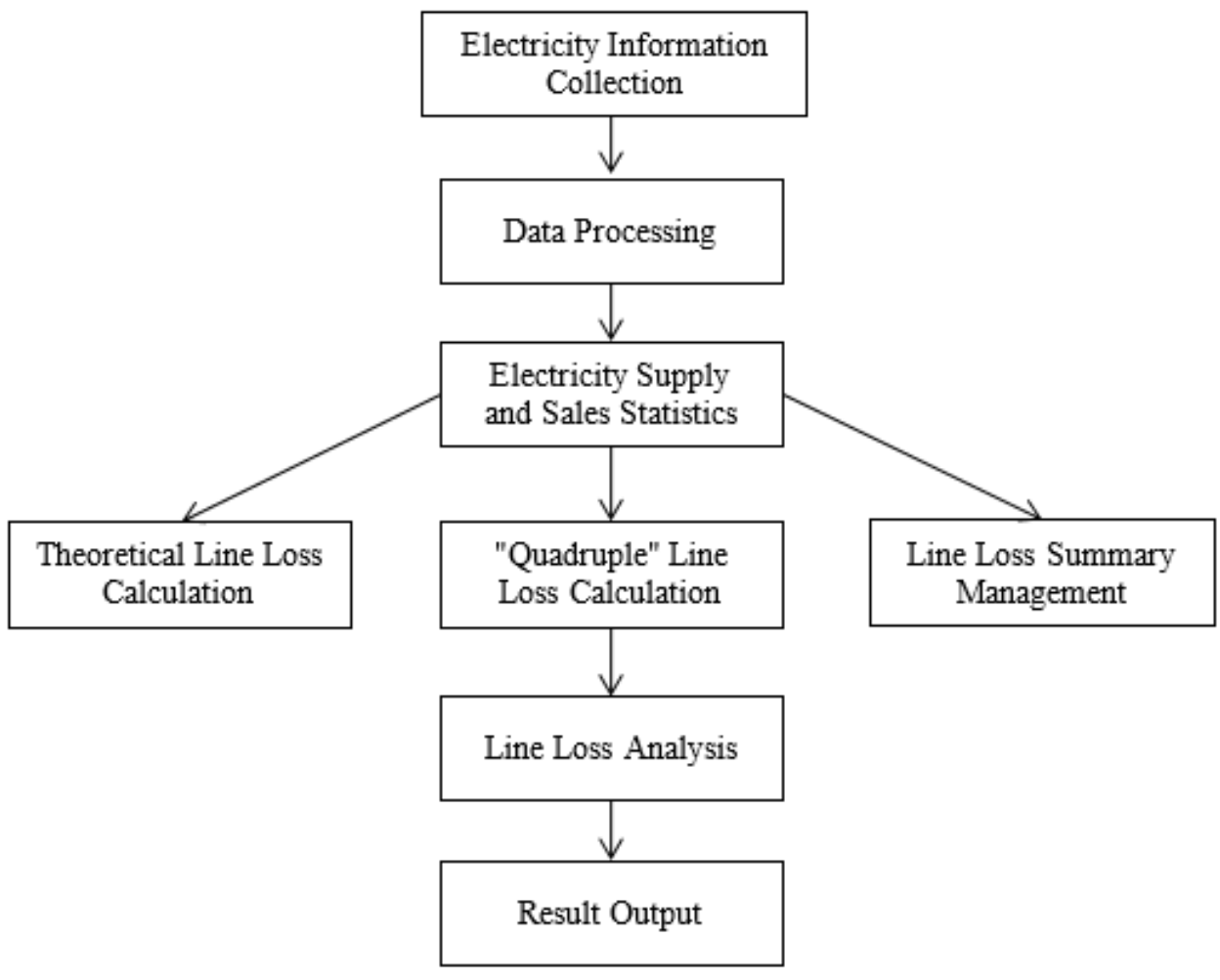

Figure 2 Line Loss Analysis Module Analysis Process

The line loss analysis module is an advanced application module in the entire management system, mainly for calling the line loss statistics and analysis and calculation of the line loss data. The analysis process of the specific line loss analysis module is shown in Figure 2.

\section{Conclusion}

Through the above design, this paper constructs a power collection and line loss analysis system that can be used in power companies by means of current mainstream computer technology and current big data processing methods. Through the system, the calculation and summarization of line losses in different periods and different regions can be realized, thereby providing a more comprehensive line loss analysis system for power companies and improving the utilization efficiency of electricity.

\section{References}

[1] Bo Zhao, Xiaojuan Tian, Zhe Sun. Application of Typical Network Communication Mode in Taiwan Area[J]. Volkswagen Power, 2015, 30(04): 28-30.

[2] Yu Tang, Bo Shao, Xunhu Wei, Hongyang Jiang, Yufei Wu. A Network-wide Topology Display System Based on Terminal Communication Access Network[J]. Communications and Information Technology, 2015(03): 92-94+98.

[3] Yu Song, Haiyan Zheng, Fei Yin. Line Loss Management Based on Intelligent Power Big Data Analysis[J]. Electric Power Information and Communication Technology, 2015, 13(08): 132-135.

[4] Wensi Huang, Yuanbin Xu, Baoping Zou, Xin Lu. Analysis of Line Loss Calculation Based on Big Data[J]. Electrical Application, 2015, 34(20): 79-84.

[5] Ruqian Song, Liu Jinliang, Fang Zhiyuan. Application of Marketing Acquisition System[J]. China Science and Technology Information, 2016(22): 16-17. 
[6] Kong Qinghua, Liu Fei, Li Jun. Jining City: “Multi-integration” Leading the Wind[J]. China Power Enterprise Management, 2017(14): 48-53.

[7] YaNan Chu. Practice and Prospect of Energy Data Acquisition Applications[J]. Electrical Applications, 2011, 30(09): 44-47. 